\title{
Time from stroke onset to groin puncture affects rate of recanalisation after mechanical thrombectomy: a real-life single centre experience
}

\author{
Klaudia Nowak, Justyna Derbisz, Jeremiasz Jagiełła, Roman Pułyk, \\ Tadeusz Popiela, Agnieszka Słowik \\ Neurology Department, Jagiellonian University, Krakow, Poland
}

\begin{abstract}
Aim of the study. We investigated whether the time elapsed between stroke onset and groin puncture (SO-GP) affects the rate of recanalisation as measured by the Thrombolysis in Cerebral Infarction (TICI) scale.

Clinical rationale for the study. There is no doubt that the effectiveness of thrombolysis in acute ischaemic stroke (AIS) is time-dependent. There is growing evidence that there is a correlation between SO-GP time and rate of recanalisation in patients treated by mechanical thrombectomy (MT).

Materials and methods. This study was performed in patients treated in the Comprehensive Stroke Centre in Krakow that covers 3.5 million inhabitants. The following data was collected for this study: demographics, stroke risk factors, transportation (directly from home or via another hospital), admission NIHSS, IV rt-PA administration prior to MT, the number of passes used during MT, and SO-GP time. The favourable outcome measure was $\mathrm{TICI} 2 \mathrm{~b}$ or 3.

Results. 223 patients (48.4\% females; mean age: $66.0 \pm 16.6$ years) with anterior circulation strokes were treated by MT; $64.6 \%$ arrived directly from home. Mean admission NIHSS was $15.6 \pm 5.3$. IV rtPA was administered in $68.6 \%$ of patients. At least two thrombectomy passes were required in $20.6 \%$ of cases. Median SO-GP time was 240 minutes (IQR range: $180-305$ minutes). Grade 3 or $2 \mathrm{~b} \mathrm{TICl}$ scores were obtained in $70.4 \%$ of patients. Univariate logistic regression showed that among all studied parameters, only NIHSS affected the rate of recanalisation, but in a multivariate logistic regression model, the only parameter that affected the rate of recanalisation was the SO-GP time (OR $=0.76 ; 95 \% \mathrm{Cl}: 0.60-0.98, \mathrm{p}=0.03)$.
\end{abstract}

Conclusions and clinical implications. We suggest that SO-GP time affects the rate of recanalisation in patients with MT.

Key words: stroke, thrombectomy, ischaemic stroke, time, recanalisation, groin puncture

(Neurol Neurochir Pol 2020; 54 (2): 156-160)

\section{Introduction}

In 2015, mechanical thrombectomy (MT) was introduced as an emergency causative treatment of ischaemic stroke (IS) [1]. Until then, intravenous (IV) rt-PA was the only evidence-based reperfusion therapy that improved clinical outcome. There is a lot of data showing that the effectiveness of IV rt-PA is time-dependent [2]. There is also growing evidence that different outcome measures in MT are also time-dependent [3-11].
We investigated whether the time elapsed between stroke onset and groin puncture (SO-GP) affected the rate of recanalisation as measured by TICI (Thrombolysis in Cerebral Infarction) criteria.

\section{Clinical rationale for the study}

There is growing evidence that in patients treated by MT there is a significant correlation between the rate of recanalisation and the SO-GP time. We looked into whether the 
SO-GP time affected the rate of recanalisation as measured by TICI criteria.

\section{Materials and methods}

We collected data from 223 patients with anterior circulation acute ischaemic stroke (AIS) selected out of 246 patients treated by MT in the Comprehensive Stroke Centre, Department of Neurology, University Hospital in Krakow between January 2013 and January 2019. The diagnosis of stroke was made according to the WHO definition.

Each patient provided signed consent or gave their oral consent in the presence of two doctors. The approval numbers of the Bioethics Committee Agreement for the collection of data are as follows: KBET/12/B/2013; KBET/178/B/2013; $\mathrm{KBET} / 54 / \mathrm{B} / 2007$.

The standardised diagnostic work-up included: demographics (age and gender); stroke risk factors (hypertension, myocardial infraction, ischaemic heart disease, atrial fibrillation, diabetes mellitus); stroke aetiology according to the Trial of Org10172 in acute Stroke Treatment (TOAST) criteria; and stroke severity on admission assessed by the National Institutes of Health Stroke Scale (NIHSS) [13].

The following data was also collected: route of transportation (i.e. directly from home or via another hospital), SO-GP time, treatment with IV rt-PA before MT, the number of passes used during the procedure, and the type of device, i.e. Solitaire Stent Retriever (Medtronic, Minneapolis, MN, USA), Penumbra Aspiration System (Penumbra Inc., Alameda, CA,
USA), Trevo Stent Retriever (Stryker Neurovascular, Fremont, CA, USA), ERIC (MicroVention Inc., Terumo), Preset (Phenox $\mathrm{GmbH}$ ), or Apeiro (Acandis $\mathrm{GmbH}$ ).

Additionally, the following immediate outcome measures were also analysed: recanalisation results according to TICI criteria: grade $0-$ no perfusion; grade $1-$ penetration with minimal perfusion; grade $2 \mathrm{a}-$ partial perfusion, $<50 \%$; $2 \mathrm{~b}-$ partial reperfusion, $\geq 50-99 \%$; grade $3-$ no flow constraint and complete perfusion [14]; and post-treatment haemorrhagic complications classified according to the ECASS-2 classification: HI-1: small petechiae, HI-2: more confluent petechiae, $\mathrm{PH}-1:<30 \%$ of the infracted area with mild space-occupying effect, $\mathrm{PH}-2>30 \%$ of the infracted area with significant space-occupying effect. Early outcome measures evaluated on discharge were: stroke severity assessed by the NIHSS and functional outcome assessed by the modified Rankin Scale (mRS) [16]. Late outcome measure was mRS at day 90 after stroke.

For the purpose of this study, the following data was analysed: demographics; stroke risk factor profile; stroke severity on admission; route of transportation; IV rtPA administration prior to MT; the number of passes used during MT; SO-GP time; and TICI score after the procedure. Successful reperfusion was defined as TICI $2 \mathrm{~b}-3$.

\section{Results}

Demographics and baseline characteristics of the included patients are presented in Table 1.

Table 1. Demographics, stroke risk factor profile and clinical data in patients with successful reperfusion ( $\mathrm{TICl} 2 \mathrm{~b} / 3$ ) compared to others (univariate analysis)

\begin{tabular}{|c|c|c|c|}
\hline & $\begin{array}{c}\mathrm{TICl}=2 \mathrm{~b}, 3 \\
(\mathrm{n}=157)\end{array}$ & $\begin{array}{c}\mathrm{TICl}=0,1,2 \mathrm{a} \\
(\mathrm{n}=66)\end{array}$ & $\mathbf{P}$ \\
\hline Age, years & $66.0 \pm 15.03$ & $66.0 \pm 13.7$ & 0.98 \\
\hline Gender, female $(n, \%)$ & $73(46.5)$ & $35(53.0)$ & 0.37 \\
\hline $\begin{array}{l}\text { Transportation (n, \%): } \\
1 \text { - directly from home } \\
2 \text { - from other hospital in Krakow } \\
3 \text { - from hospital outside Krakow }\end{array}$ & $\begin{array}{l}1=98(62.4) \\
2=24(15.3) \\
3=35(22.3)\end{array}$ & $\begin{array}{l}1=46(70.0) \\
2=12(18.2) \\
3=18(12.1)\end{array}$ & 0.21 \\
\hline $\begin{array}{l}\text { Number of devices } \\
n=1(n, \%) \\
n>1\end{array}$ & $\begin{array}{c}104(66.2) \\
34(21.6)\end{array}$ & $\begin{array}{l}54(81.8) \\
12(18.2)\end{array}$ & 0.3 \\
\hline NIHSS on admission (mean \pm SD) & $15.2 \pm 5.3$ & $16.7 \pm 5.2$ & 0.051 \\
\hline Hypertension $(n, \%)$ & $111(70.7)$ & $50(75.6)$ & 0.44 \\
\hline Diabetes mellitus $(n, \%)$ & $41(26.1)$ & $19(28.8)$ & 0.68 \\
\hline Ischaemic heart disease $(n, \%)$ & $44(28.0)$ & $20(30.3)$ & 0.73 \\
\hline Atrial fibrillation $(n, \%)$ & $65(41.4)$ & $25(37.9)$ & 0.62 \\
\hline SO-GP time, minutes (minimum, maximum) & $\begin{array}{c}247.3 \pm 88.6 \\
(93-600)\end{array}$ & $265 \pm 86.0(150-600)$ & 0.16 \\
\hline Treatment with IV rt-Pa before MT ( $n, \%)$ & $104(66.2)$ & $49(74.2)$ & 0.24 \\
\hline
\end{tabular}

NIHSS - National Institutes of Health Stroke Scale; SO-GP time - time from stroke onset to groin puncture; IV rt-Pa - intravenous recombinant tissue plasminogen activator; MT — mechanical thrombectomy 
Table 2. Factors affecting rate of recanalisation as measured by $\mathrm{TICl}$ score (multivariate analysis)

\begin{tabular}{lccc} 
& \multicolumn{3}{c}{ Multivariate analysis } \\
& OR & $\mathbf{9 5 \%} \mathbf{~ C l}$ & $\mathbf{P}$ \\
\hline Age, years & 1.01 & $0.99-1.04$ & 0.3 \\
Gender, female & 0.68 & $0.35-1.29$ & 0.23 \\
$\begin{array}{l}\text { Transportation from another stroke } \\
\text { unit }\end{array}$ & 2.11 & $0.98-4.53$ & 0.06 \\
Number of devices & 1.37 & $0.63-2.98$ & 0.43 \\
NIHSS on admission & 0.95 & $0.89-1.01$ & 0.11 \\
Hypertension & 0.64 & $0.28-1.43$ & 0.27 \\
Diabetes mellitus & 1.01 & $0.5-2.05$ & 0.97 \\
Ischaemic heart disease & 0.91 & $0.45-1.84$ & 0.78 \\
Atrial fibrillation & 1.19 & $0.59-2.43$ & 0.62 \\
SO-GP time, hours & 0.77 & $0.6-0.98$ & 0.04 \\
Treatment with IV rt-PA prior MT & 0.77 & $0.39-1.54$ & 0.46
\end{tabular}

NIHSS - National Institutes of Health Stroke Scale; SO-GP time - time from stroke onset to groin puncture; IV rt-Pa - intravenous recombinant tissue plasminogen activator; MT — mechanical thrombectomy

Median SO-GP time was 240 minutes (IQR range: 180 305 minutes). Grade 3 or $2 \mathrm{~b}$ TICI scores were obtained by $70.4 \%$ of patients. Univariate logistic regression showed that among all studied parameters, only the NIHSS score affected the rate of recanalisation ( $\mathrm{p}=0.051$ ), but a multivariate logistic regression model that included all analysed data showed that the only parameter that affected the rate of recanalisation was the SO-GP time (OR $=0.76 ; 95 \%$ CI: 0.60-0.98, $\mathrm{p}=0.04)(\mathrm{Tab} .2)$.

Of the 223 presented patients, $120(53.8 \%)$ were treated by IV rt-PA up to 3 hours, $24(10.8 \%)-3-4.5$ hours, and 79 (35.4\%) had no IV rt-PA administration prior to MT. Treatment with IV rt-PA up to 3 hours after stroke onset did not affect recanalisation $(\mathrm{OR}=1.14 ; 95 \% \mathrm{CI}=0.58-2,25)$.

\section{Discussion}

We have shown that the rate of recanalisation after MT depends on the SO-GP time and is not affected by demographics, stroke risk factors, stroke severity on admission, route of transportation, IV rtPA administration prior to MT, or number of passes during MT.

This is an important finding because SO-GP time lapse is a modifiable factor affecting outcome and efforts should be made to shorten it as much as possible. Studies show that there are only a few modifiable factors that affect successful recanalisation after MT. For example, Ozdemir et al. showed that the number of passes during the procedure correlated with poorer recanalisation [9]. They also showed that a larger clot extent and proximal clot location causing resistant clots may explain the need for more passes [9].

The correlation between SO-GP time and recanalisation $[3,7]$ or different clinical outcome $[5,18,7,9,20]$ measures was analysed in different cohorts of AIS patients treated by MT. In two studies, the outcome was measured by the TICI score [3, 7] after the procedure. Hassan et al. presented similar findings to ours, showing that SO-GP time is lower in patients with favourable TICI outcomes [7]. In contrast, Bourcier et al. [3], who conducted a meta-analysis of individual patient data from seven randomised trials of the Highly Effective Reperfusion Using Multiple Endovascular Devices (HERMES) group did not show a correlation between SO-GP time and TICI score. However, they showed that TICI score was affected by the time between admission to hospital and groin puncture (A-GP) or the time of imaging to groin puncture (I-GP). There is no doubt that at present the time of admission and the time of imaging, in contrast to the time of disease onset, are precisely documented by hospital IT systems. On the other hand, determining the exact time of a stroke is difficult due to the frequent absence of witnesses, the possibility or not of verbal contact with the patient, and so on..

Five other studies have evaluated the influence of SO-GP time on $\mathrm{mRS}$ at day $90[5,7,9,18,20]$ or all-cause mortality at day $90[18,20]$. Three of these showed a significant correlation between the two parameters - the shorter the SO-GP, the better the clinical outcome $[7,18,20]$. Two other studies did not show such a correlation $[9,5]$.

A number of studies have measured different time intervals that may affect early reperfusion or clinical outcomes in AIS patients treated by MT i.e. A-GP time, I-GP time, time between initial brain imaging and recanalisation (I-R), procedural time (groin puncture to reperfusion time; GP-R time), or time from stroke onset to recanalisation (SO-R time).

Interestingly, most studies on this topic have analysed the time elapsed between SO-R. All showed significant correlations between shorter SO-R and different clinical outcome measures, both short-term (intracranial haemorrhage rate 24 hours after the procedure $[7,20])$ and long-term (mRS at day $90[5-11,20]$, or mortality at day $90[11,20])$.

This approach, compared to ours, seems to be more logical in respect to stroke pathophysiology; it evaluates time to the beginning to recanalisation. It should be highlighted that nowadays in most hospitals the time of performing different procedures is documented automatically and precisely, while the time of stroke onset is less reliable. Unfortunately, we were not able to collect retrospectively the durations of the procedures in the studied group of patients.

Three studies $[4,5,7]$ that focused on analysing GP-R time showed that this time lapse affects recanalisation as measured by TICI scale $[4,7]$ or mRS $[4,5,7]$. Interestingly, they revealed that the duration of the procedure can vary significantly; the shortest noted procedure was 40.7 minutes while the longest was 134.3 minutes.

Other papers have shown that successful recanalisation (mTICI $2 \mathrm{~b} / 3$ ) or a good clinical outcome ( $\mathrm{mRS} 0-2$ at day 90 [6, $8,18-20])$ were also associated with shorter I-R [6, 8], I-GP $[3,19]$ or A-GP [18], times. These findings highlight also the need for urgency after a patient's admission to hospital and 
the need to consider new strategies to reduce this time lapse between interhospital transfers [19].

Stroke is a devastating disease in which aetiological treatment is available only for a small percentage of patients. Only a few of them profit from this treatment; within a therapeutic window NNT for IV rt-PA $=14$ and NNT for MT $=3$.

That is why identifying all factors affecting outcome, and analysing their significance, is so important to improve the quality of care for stroke patients.

\section{Conclusions}

In this analysis, based on a real-life single centre experience, we suggest that SO-GP time may affect the rate of recanalisation in patients with MT. However, based on the literature, we recommend also the measurement of other milestone times significant in acute stroke care, especially the time to recanalisation.

\section{Clinical implications/future directions}

Time-lapse between stroke onset and groin puncture affects the rate of recanalisation in AIS with MT. This is an important finding because SO-GP time lapse is a modifiable factor affecting outcome and all efforts should be made to shorten it as much as possible.

Funding: This publication was prepared without any external source of funding

Conflicts of interest: The authors declare no potential conflict of interest with respect to the research, authorship, and/ or publication of this article

\section{References:}

1. Lambrinos A, Schaink AK, Dhalla I, et al. Mechanical Thrombectomy in Acute Ischemic Stroke: A Systematic Review. Can J Neurol Sci. 2016; 43(4): 455-460, doi: 10.1017/cjn.2016.30, indexed in Pubmed: 27071728.

2. Alcock S, Sawatzky JAV. "Time is Brain:" A concept anaiysis. Can J Neurosci Nurs. 2016; 38(2): 5-11, indexed in Pubmed: 29465169.

3. Bourcier R, Goyal M, Liebeskind DS, et al. HERMES Trialists Collaboration. Association of Time From Stroke Onset to Groin Puncture With Quality of Reperfusion After Mechanical Thrombectomy: A Meta-analysis of Individual Patient Data From 7 Randomized Clinical Trials. JAMA Neurol. 2019; 76(4): 405-411, doi: 10.1001/jamaneurol.2018.4510, indexed in Pubmed: 30667465.

4. Spiotta AM, Vargas J, Turner R, et al. The golden hour of stroke intervention: effect of thrombectomy procedural time in acute ischemic stroke on outcome. J Neurointerv Surg. 2014; 6(7): 511-516, doi: 10.1136/neurintsurg-2013-010726, indexed in Pubmed: 24014466.
5. Fransen P, Berkhemer O, Lingsma H, et al. Time to Reperfusion and Treatment Effect for Acute Ischemic Stroke. JAMA Neurology. 2016; 73(2): 190, doi: 10.1001/jamaneurol.2015.3886.

6. Ribo M, Molina CA, Cobo E, et al. REVASCAT Trial Investigators. Association Between Time to Reperfusion and Outcome Is Primarily Driven by the Time From Imaging to Reperfusion. Stroke. 2016; 47(4): 999-1004, doi: 10.1161/STROKEAHA.115.011721, indexed in Pubmed: 26956258.

7. Hassan AE, Shariff U, Saver JL, et al. Impact of procedural time on clinical and angiographic outcomes in patients with acute ischemic stroke receiving endovascular treatment. J Neurointerv Surg. 2019; 11(10): 984-988, doi: 10.1136/neurintsurg-2018-014576, indexed in Pubmed: 30842302.

8. Goyal M, Jadhav AP, Bonafe A, et al. SWIFT PRIME investigators. Analysis of Workflow and Time to Treatment and the Effects on Outcome in Endovascular Treatment of Acute Ischemic Stroke: Results from the SWIFT PRIME Randomized Controlled Trial. Radiology. 2016; 279(3): 888-897, doi: 10.1148/radiol.2016160204, indexed in Pubmed: 27092472 .

9. Ozdemir O, Giray S, Arlier Z, et al. Predictors of a Good Outcome after Endovascular Stroke Treatment with Stent Retrievers. ScientificWorldJournal. 2015; 2015: 403726, doi: 10.1155/2015/403726, indexed in Pubmed: 26137591.

10. Khatri P, Yeatts SD, Mazighi M, et al. IMS III Trialists. Time to angiographic reperfusion and clinical outcome after acute ischaemic stroke: an analysis of data from the Interventional Management of Stroke (IMS III) phase 3 trial. Lancet Neurol. 2014; 13(6): 567-574, doi: $10.1016 /$ S1474-4422(14)70066-3, indexed in Pubmed: 24784550.

11. Nogueira RG, Smith WS, Sung G, et al. MERCI and Multi MERCI Writing Committee. Effect of time to reperfusion on clinical outcome of anterior circulation strokes treated with thrombectomy: pooled analysis of the MERCI and Multi MERCI trials. Stroke. 2011; 42(11): 3144-3149, doi: 10.1161/STROKEAHA.111.624163, indexed in Pubmed: 21921278.

12. Starostka-Tatar A, Łabuz-Roszak B, Skrzypek M, et al. [Definition and treatment of stroke over the centuries]. Wiad Lek. 2017; 70(5): 982987, indexed in Pubmed: 29203753.

13. Rödén-Jüllig $A$, Britton M, Gustafsson C, et al. Validation of four scales for the acute stage of stroke. J Intern Med. 1994; 236(2): 125-136, doi: 10.1111/j.1365-2796.1994.tb01274.x, indexed in Pubmed: 8046311.

14. Fugate JE, Klunder AM, Kallmes DF. What is meant by “TICI”? AJNR Am J Neuroradiol. 2013; 34(9): 1792-1797, doi: 10.3174/ajnr.A3496, indexed in Pubmed: 23578670.

15. Neuberger U, Möhlenbruch MA, Herweh C, et al. Classification of Bleeding Events: Comparison of ECASS III (European Cooperative Acute Stroke Study) and the New Heidelberg Bleeding Classification. Stroke. 2017; 48(7): 1983-1985, doi: 10.1161/STROKEAHA.117.016735, indexed in Pubmed: 28455322.

16. Sulter G, Steen C, De Keyser J. Use of the Barthel index and modified Rankin scale in acute stroke trials. Stroke. 1999; 30(8): 1538-1541, doi: 10.1161/01.str.30.8.1538, indexed in Pubmed: 10436097.

17. Yaghi S, Willey JZ, Cucchiara B, et al. American Heart Association Stroke Council; Council on Cardiovascular and Stroke Nursing; Council on Clinical Cardiology; and Council on Quality of Care and Outcomes Research. Treatment and Outcome of Hemorrhagic Transformation 
After Intravenous Alteplase in Acute Ischemic Stroke: A Scientific Statement for Healthcare Professionals From the American Heart Association/American Stroke Association. Stroke. 2017; 48(12): e343-e361, doi: 10.1161/STR.0000000000000152, indexed in Pubmed: 29097489.

18. Sun CHJ, Ribo M, Goyal M, et al. Door-to-puncture: a practical metric for capturing and enhancing system processes associated with endovascular stroke care, preliminary results from the rapid reperfusion registry. J Am Heart Assoc. 2014; 3(2): e000859, doi: 10.1161/ jaha.114.000859, indexed in Pubmed: 24772523.
19. Sun CHJ, Nogueira RG, Glenn BA, et al. "Picture to puncture": a novel time metric to enhance outcomes in patients transferred for endovascular reperfusion in acute ischemic stroke. Circulation. 2013; 127(10): 1139-1148, doi: 10.1161/CIRCULATIONAHA.112.000506, indexed in Pubmed: 23393011.

20. Saver JL, Goyal M, van der Lugt A, et al. HERMES Collaborators. Time to Treatment With Endovascular Thrombectomy and Outcomes From Ischemic Stroke: A Meta-analysis. JAMA. 2016; 316(12): 1279-1288, doi: 10.1001/jama.2016.13647, indexed in Pubmed: 27673305. 